\title{
Criminologie
}

\section{Regards de Nunavimmiuts sur les raisons de la consommation et ses effets}

\author{
Natacha Brunelle, Chantal Plourde, Michel Landry et Annie Gendron
}

Volume 42, numéro 2, automne-hiver 2009

Régulations sociopénales et peuples autochtones

URI : https://id.erudit.org/iderudit/038597ar

DOI : https://doi.org/10.7202/038597ar

Aller au sommaire du numéro

\section{Éditeur(s)}

Les Presses de l'Université de Montréal

ISSN

0316-0041 (imprimé)

1492-1367 (numérique)

Découvrir la revue

Citer cet article

Brunelle, N., Plourde, C., Landry, M. \& Gendron, A. (2009). Regards de Nunavimmiuts sur les raisons de la consommation et ses effets. Criminologie, 42(2), 9-29. https://doi.org/10.7202/038597ar
Résumé de l'article

Dans le présent article sont exposés les résultats d'une étude sur la consommation de substances psychoactives au Nunavik. Le volet qualitatif de cette étude comporte 108 entretiens semi-dirigés auprès de jeunes, de parents, de leaders et d'Aînés de quatre villages. L'analyse de contenu thématique des résultats a mis en lumière différentes raisons et différents effets de la consommation au Nunavik du point de vue des participants. Parmi les raisons évoquées par les interviewés, on retrouve le plaisir, l'ennui et le manque d'activités, la « colonisation ", l'adaptation (coping) à divers problèmes (pauvreté, abus, suicide) et la transmission intergénérationnelle. Sur la question des conséquences de la consommation, les effets sur la famille, dont la violence conjugale et la négligence envers les enfants, mais aussi les comportements déviants, incluant la conduite avec capacités affaiblies, les voies de fait et les délits lucratifs ont été évoqués. Ces résultats sont discutés à la lumière des connaissances actuelles sur les causes et conséquences de la consommation chez les Inuits et les non-Inuits. 


\title{
Regards de Nunavimmiuts sur les raisons de la consommation et ses effets
}

\author{
Natacha Brunelle \\ Titulaire, Chaire de recherche du Canada (CRC) sur les trajectoires d'usage de drogues et les \\ problématiques associées \\ Chercheure, Institut universitaire sur les dépendances, RISQ et GRIAPS \\ Professeure, Département de psychoéducation, Université du Québec à Trois-Rivières (UQTR) \\ Natacha.brunelle@uqtr.ca \\ Chantal Plourde \\ Chercheure, RISQ et CICC-UQTR \\ Professeure, Département de psychoéducation, UQTR \\ Chantal.plourde@uqtr.ca \\ Michel Landry \\ Co-directeur, RISQ \\ Chercheur, RISQ et Institut universitaire sur les dépendances \\ michel.landry@ssss.gouv.qc.ca

\section{Annie Gendron} \\ Coordonnatrice, CRC sur les trajectoires d'usage de drogues et les problématiques associées \\ Département de psychoéducation, UQTR \\ Annie.gendron@uqtr.ca
}

RÉSUMÉ - Dans le présent article sont exposés les résultats d'une étude sur la consommation de substances psychoactives au Nunavik. Le volet qualitatif de cette étude comporte 108 entretiens semi-dirigés auprès de jeunes, de parents, de leaders et d'Aînés de quatre villages. L'analyse de contenu thématique des résultats a mis en lumière différentes raisons et différents effets de la consommation au Nunavik du point de vue des participants. Parmi les raisons évoquées par les interviewés, on retrouve le plaisir, l'ennui et le manque d'activités, la "colonisation», l'adaptation (coping) à divers problèmes (pauvreté, abus, suicide) et la transmission intergénérationnelle. Sur la question des conséquences de la consommation, les effets sur la famille, dont la violence conjugale et la négligence envers les enfants, mais aussi les comportements déviants, incluant la conduite avec capacités affaiblies, les voies de fait et les délits lucratifs ont été évoqués. Ces résultats sont discutés à la lumière des connaissances actuelles sur les causes et conséquences de la consommation chez les Inuits et les non-Inuits.

MOTS-CLÉS - Drogue, alcool, Inuit, Nunavik, Québec, recherche qualitative. 


\section{Introduction}

Le Nunavik est situé au nord du $55^{\mathrm{e}}$ parallèle et représente le tiers de la superficie totale du Québec (Statistique Canada, 2006). Il forme un territoire composé d'environ 9000 personnes réparties dans 14 villages bordant la baie d'Ungava et la baie d'Hudson (Régie régionale de la santé et des services sociaux du Nunavik, 2004-2005).

En 2000, des leaders de certains de ces villages ainsi que la Régie régionale de la santé et des services sociaux du Nunavik ont manifesté le besoin qu'une étude soit conduite pour faire état de la consommation de substances psychoactives $\left(\mathrm{SPA}^{1}\right)$ au Nunavik. L'étude de recension menée par Mercier et al. (2002) a démontré clairement qu'il y avait chez les Inuit du Nunavik une préoccupation face à la consommation de SPA et à ses effets sur le bien-être physique, psychologique, social et culturel des Nunavimmiuts ${ }^{2}$. La consommation chez les jeunes Inuits suscitait particulièrement l'inquiétude. L'absence de données empiriques et récentes qui rendraient compte à la fois de l'ampleur du problème de consommation et de la signification de celle-ci dans la population représente un enjeu fondamental auquel deux études ont tenté de pallier ces dernières années. L'Institut national de santé publique du Québec (INSPQ) a conduit une vaste enquête en 2004 sur la santé des habitants du Nunavik âgés de 15 ans et plus. Parmi les résultats, on observe que la proportion de consommateurs occasionnels ou réguliers d'alcool y est plus basse (77\%) qu'ailleurs au Québec (85\%), alors que la proportion de consommateurs excessifs («binge drinkers») y est plus élevée (24\% vs 7,5\%). Par ailleurs, environ $60 \%$ des participants rapportent avoir consommé au moins une drogue autre que l'alcool dans la dernière année, généralement du cannabis. Cette proportion est quatre fois plus élevée qu'ailleurs au Canada (Anctil, 2008). Mais cette étude de l'INSPQ ne fournit pas de portrait plus spécifique de la consommation chez les adolescents du Nunavik, pas plus que d'informations sur le point de vue des Nunavimmiuts concernant les contextes de consommation, les motivations et conséquences de celle-ci. Or, c'est ce à quoi la présente étude s'attarde. Le portrait de consommation chez les adolescents concerne le volet statistique de notre étude dont les résultats

1. Le terme SPA englobe l'alcool et les autres drogues.

2. Le terme Nunavimmiut fait ici référence aux personnes qui habitent le Nunavik, personnes majoritairement d'origine ethnique inuite. 
sont présentés dans d'autres écrits (Plourde et al., 2007; Brunelle et al., sous presse). L'objet de cet article concerne le volet qualitatif de l'étude et consiste à rapporter et à organiser le plus fidèlement possible les points de vue des Nunavimmiuts sur les raisons et les conséquences de la consommation de SPA au Nunavik.

\section{Méthode}

Il est connu que l'engagement et la participation des Autochtones et Inuits constituent un gage d'assurance du réalisme et de la faisabilité d'un projet de recherche (Fletcher et Hunter, 2003; Greabell et al., 2005). Dans cet esprit, un comité consultatif a donc été créé avant même le dépôt de la demande de subvention aux Instituts de recherche en santé du Canada (IRSC), qui était composé d'une dizaine d'acteurs clés travaillant sur les deux côtes du Nunavik. Ce comité s'est réuni à quatre reprises entre le moment de l'élaboration du projet de recherche et celui de la diffusion des résultats. L'approche privilégiée tout au long du processus a été une recherche collaborative (Dufort et al., 2005).

\section{Les participants}

L'échantillon du volet qualitatif de l'étude se compose de 109 participants ( 59 femmes et 50 hommes) issus de quatre villages du Nunavik. Les villages ont été choisis par le comité consultatif sur la base de leur localisation géographique (deux en bordure de la baie d'Ungava et deux en bordure de la baie d'Hudson) et de leur densité démographique (un petit et un grand village sur chaque côte). Le tableau 1 présente les données sociodémographiques des répondants selon leur catégorie d'appartenance. Le comité consultatif et les chercheurs trouvaient très important de diversifier l'échantillon en fonction de catégories de répondants (variables stratégiques pour l'échantillonnage). Parmi ces catégories figure celle de décrocheurs, terme par lequel nous désignons les jeunes de 18 ans et moins qui ne fréquentaient pas l'école durant la période de collecte de données et qui n'avaient pas l'intention d'y retourner, du moins pas à court terme. À son tour, le terme leaders fait généralement référence aux personnes membres d'un conseil municipal ou d'un organisme inuit (gouvernemental ou non). Quant au terme Aînés, il fait référence aux personnes considérées comme des sages dans le village, sages auprès de qui plusieurs demandent conseil. Il s'agit 
généralement de personnes de 50 ans et plus. Il est à noter qu'elles peuvent aussi faire partie des conseils municipaux, mais c'est leur statut d'Aîné qui prévaut sur celui de leader dans notre classification. Somme toute, le point de vue des adultes (parents, leaders et Aînés : $\mathrm{n}=69$ ) est davantage représenté que celui des jeunes (élèves et décrocheurs: $\mathrm{n}=$ 40) dans le cadre du volet qualitatif de l'étude.

TA B LEA U 1

Répartition des participants

\begin{tabular}{l|cccc}
\hline & $\boldsymbol{N}$ & $\begin{array}{c}\text { Genre } \\
\text { femme/homme }\end{array}$ & Âge moyen & $\begin{array}{c}\hat{\text { Age }} \\
\text { minimum/maximum }\end{array}$ \\
\hline Jeunes élèves & 23 & $8 \mathrm{H} / 15 \mathrm{~F}$ & 15,6 ans & 14 à 18 ans \\
Décrocheurs & 17 & $6 \mathrm{H} / 11 \mathrm{~F}$ & 16,2 ans & 14 à 18 ans \\
Parents & 24 & $10 \mathrm{H} / 14 \mathrm{~F}$ & 36,7 ans & 21 à 66 ans \\
Leaders & 22 & $13 \mathrm{H} / 9 \mathrm{~F}$ & 46,1 ans & 25 à 60 ans \\
Aînés & 23 & $13 \mathrm{H} / 10 \mathrm{~F}$ & 72,1 ans & 55 à 94 ans \\
\hline
\end{tabular}

\section{Les entretiens}

L'entretien de recherche qualitatif semi-dirigé (Poupart et al., 1997) s'est fait avec les 109 répondants à partir d'un guide d'entretien comportant essentiellement des questions ouvertes sur les contextes, motivations et effets de la consommation sur les plans : 1) communautaire; 2) familial; et 3) personnel. Les dernières questions portent sur les solutions envisagées pour améliorer la situation (les résultats spécifiques à ces solutions sont traités dans Plourde et al. (soumis). Les entretiens se sont déroulés en anglais, sauf une trentaine en inuktitut avec interprète et de rares fois en français. Nous avons effectué 108 entretiens, puisqu'un entretien a été conduit avec deux personnes en même temps.

\section{La méthode d'analyse}

La phénoménologie scientifique telle que définie par Giorgi (1997) a orienté l'analyse, dont l'étape de la réduction du matériel. Pour ce faire, Giorgi affirme que le chercheur doit mettre de côté les connaissances qu'il a déjà sur le sujet à l'étude pour se concentrer entièrement sur l'expérience concrète de la personne interrogée (Giorgi, 1997). En fin de processus, le chercheur pourra affirmer que l'expérience concrète qu'il a recueillie auprès des principaux intéressés correspond au vécu 
même de ces personnes et non à un compte rendu objectif de ce qui s'est passé en vérité. La méthode de réduction de l'imposant corpus de verbatim s'est faite à travers l'analyse de contenu thématique (Deslauriers, 1991) et, plus particulièrement, par thématisation séquenciée (Paillé et Mucchielli, 2003). Une analyse verticale de chacun des entretiens a été faite par une analyse transversale afin de dégager les tendances observables dans les propos des répondants (Deslauriers, 1991). Mentionnons que quatre délégués inuits (deux adultes et deux jeunes adultes) ont participé aux analyses qualitatives, ce qui s'inscrit dans la démarche de recherche collaborative adoptée.

Nous avons effectué une analyse du contenu manifeste des entretiens. Ainsi, nous avons tenté de respecter le plus fidèlement possible les propos et points de vue des Nunavimmiuts quant à l'usage de SPA, et ce, sans tomber dans ce qui aurait pu devenir un «piège intellectuel» occultant le sens profond, voire inconscient, des propos des gens. En ce sens, Giorgi (1997) décrit la conscience comme un objet de la phénoménologie. Par les différentes modes, formes ou styles qu'elle prend, la conscience ne présente pas seulement les objets de façon neutre, mais leur donne en plus une signification. La présentation des résultats considère le contenu manifeste obtenu dans les entretiens, tout en l'organisant et en le confrontant aux résultats obtenus par d'autres auteurs.

\section{Raisons de la consommation de SPA au Nunavik}

Principalement, les jeunes répondants ${ }^{3}$ ont expliqué la consommation de SPA au Nunavik par le plaisir, l'ennui/manque d'activités, l'adaptation (coping) à divers problèmes et l'influence des pairs. Quant aux adultes, ils ont davantage évoqué l'histoire ou la «colonisation» par les Blancs (traumatismes et perte d'identité), l'ennui/manque d'activités, l'influence des pairs et la transmission intergénérationnelle. Nous préciserons dans le texte lorsqu'une raison aura été davantage évoquée par les jeunes ou les adultes. Autrement, nous utiliserons l'appellation répondants.

Les motivations des Inuits à consommer ne diffèrent pas tellement de celles des non-Inuits, mais certaines de ces motivations sont davantage

3. Ici, nous avons regroupé les élèves et les décrocheurs dans la catégorie «jeunes» et nous avons également regroupé les parents, leaders et Aînés en une catégorie «adultes». Les points de divergence analysés dans les discours nous ont paru ressortir surtout en fonction du groupe d'âge. Aussi, les normes de la revue (6000 mots maximum) ne nous permettaient pas de fournir des extraits pour chaque catégorie de répondants. 
évoquées par les Nunavimmiuts que par les habitants du sud du Québec. À l'instar des autres Québécois (Brunelle et al., 2005), le plaisir est au centre des motivations pour consommer pour plusieurs répondants, jeunes et moins jeunes:

Juste pour le fun. On ne boit pas tant que ça. On boit juste pour le feeling. (Janice, jeune, 16 ans)

Je ne crois pas que j'avais réellement de motivation... [rires]. Je voulais juste l'essayer, l'expérimenter... (Kristin, jeune, 14 ans)

C'est le contexte géographique, politico-historique et sociopolitique du Nunavik qui fait probablement ressortir davantage que chez d'autres populations des motivations telles que l'ennui et le manque d'activités, la colonisation et l'adaptation (coping) à des situations adverses, dont le suicide d'un proche.

L'ennui est un thème qui a été spontanément abordé par toutes les catégories de répondants de notre étude. Ces derniers évoquent très souvent le manque de choix ou de variété dans les activités auxquelles les Nunavimmiuts peuvent se livrer. Celles disponibles (ex.: chasse et pêche) ne semblent pas suffisantes pour satisfaire les attentes des résidents du Nunavik, particulièrement les jeunes:

Nous devrions avoir plus d'activités dans ce village et plus de choses à faire, des places où nous pourrions aller pour «bang out», comme jouer au billard et des jeux et des choses... je pense que c'est pourquoi les jeunes commencent à prendre de la drogue et de l'alcool, les jeunes. (Judy, décrocheuse, 16 ans)

Chez les adultes également, l'utilisation des SPA est clairement nommée comme une échappatoire à l'ennui provoqué par un manque d'activités ou une situation de manque d'emploi. Dans quelques témoignages, c'est l'état de sobriété qui est jugé ennuyant: «Comme quand je, quand je suis normale [non intoxiquée], c'est comme je m'ennuie ou que je manque quelque chose» (Maia, parent, 35 ans). Huot (2007) a aussi mis en lumière cette réalité inuite en tant que cause de la consommation, particulièrement chez les jeunes. Tel qu'explicité, le développement des nouvelles technologies de communication donne accès à tout ce qui accompagne le mode de vie des sociétés industrialisées, accentuant davantage le décalage entre les cultures. La sédentarité imposée par les Blancs a laissé le peuple inuit aux prises avec une redéfinition de ses us et coutumes. La «colonisation» et ses effets sont d'ailleurs une grande 
préoccupation pour les Inuits, ce qui ressort surtout du discours des adultes dans notre étude.

L'un des événements les plus marquants relevés dans le discours des répondants fut l'abattage des chiens des Inuits par la GRC entre les années 1950 et 1970 . Commencée vers le milieu du XIX ${ }^{e}$ siècle avec l'implantation de services médicaux et scolaires dans le Nord par le gouvernement canadien, la sédentarisation s'intensifie et se généralise dans les années 1960-1970, période pendant laquelle, selon les répondants, les problèmes de consommation se manifestent: "Cela a commencé en 1960... Avant cela, il n'y en avait pas beaucoup [de l'alcool]» (Larry, leader, 45 ans).

Le bouleversement du mode de vie inuit (certains parlent même de «choc culturel») s'accompagne et s'intensifie par la rupture de la transmission du savoir traditionnel d'une génération à l'autre. La scolarisation des enfants, en effet, a éloigné ces derniers de leur famille, et, avec le savoir, c'est également l'intérêt pour les activités traditionnelles quotidiennes qui s'est perdu:

Vous savez, je suis allée à l'école, donc je n'ai pas appris comment coudre. Je n'ai pas appris comment faire une bordure, vous savez, de la manière traditionnelle. Je n'ai pas appris comment nettoyer la peau de phoque et le transformer $[\ldots]$ et les jeunes n'y voient aucun intérêt. (Leslie, leader, 52 ans)

Comprenons que ce n'est pas seulement le mode de vie qui est bouleversé. Toute une façon de penser, de vivre va s'imposer aux Inuits. La sédentarisation est synonyme pour certains répondants de consommation abusive, d'abus, de traumatismes et d'aliénation. Les Inuits ont été et sont encore confrontés à ce processus d'adaptation:

Mon père, il a 53 ans, et il est né dans un igloo. Donc on parle d'une vie avec quelques groupes familiaux, mettons cinq-six familles nomades qui se promènent avec les troupeaux et avec les saisons et qui sont libres comme l'air, et là du jour au lendemain, vraiment du jour au lendemain, ils ont des maisons, faut qu'ils restent dans le même village. Mais il faut dire qu'il y a plusieurs personnes qui ont réussi à s'ajuster et à s'adapter, mais il y en a beaucoup d'autres qui n'ont pas réussi. Je pense que c'est le groupe qui n'a pas réussi à faire des ajustements, à faire des différents styles de vie qui consomment le plus. (Lena, leader, 27 ans)

D'autres auteurs (Wexler, 2006; Saewyc, 2007) mentionnent que la colonisation est une piste d'explication des risques accrus d'usage et 
d'abus de SPA dans les villages inuits, métis et des Premières Nations. Mais des auteurs comme Shaggers et Gray (2002) critiquent cette conception de la culture inuite selon laquelle les individus qui en font partie n'ont aucune autre stratégie d'adaptation aux changements politiques et sociaux que l'abus de substances. Quoi qu'il en soit, la consommation en tant que stratégie d'adaptation ressort effectivement beaucoup des entretiens. La consommation comme stratégie d'adaptation est la cause majoritairement évoquée et perçue par les répondants jeunes et adultes comme expliquant la consommation abusive de SPA. C'est donc d'une certaine façon la recherche d'un mieux-être qui s'envisage dans la polarité mal-être-mieux-être. Les répondants comprennent ici les effets de la consommation comme un camouflage de douleurs psychologiques ressenties, un besoin de se «déconnecter» de la réalité, peu importe la qualité de la substance disponible:

Ça réduit la douleur quand je bois, vous savez, je peux prétendre qu'il n'y a pas de douleur. L'alcool me donne de l'espoir, du bonheur, et je sais que la douleur va être là quand je serai sobre. (Marra, parent, 36 ans)

L'expression «oublier les problèmes» ou «noyer la douleur» est le fil rouge des causes qui expliquent la consommation problématique de SPA selon les répondants. Ces expressions recouvrent une panoplie de réalités telles que la pauvreté, les problèmes familiaux, les abus physiques et sexuels et le suicide d'un proche. Tout d'abord, lorsqu'ils évoquent les problèmes familiaux, ils font référence à la violence familiale ou conjugale, à une rupture à l'intérieur d'un couple ou encore à un manque d'attention accordée aux enfants. Autant de réalités qui, selon un grand nombre de répondants, peuvent amener à une consommation abusive de SPA:

Ma mère et mon père sont divorcés maintenant. Ils ne vivent pas ensemble. Je suis contente de ça parce que ma mère se faisait battre beaucoup. [...] Il la frappait beaucoup quand il était ivre. (Rosa, jeune, 16 ans)

Les répondants désignent fréquemment comme "problème» les expériences de victimisation, notamment sous la forme des abus physiques, psychologiques ou sexuels. Les consommateurs qui en sont victimes parlent souvent de cet événement (parfois vécu à plusieurs reprises) comme d'un fait marquant, voir déclencheur, dans leur parcours de consommation:

Parce que j'ai été abusé sexuellement par une femme. Quand je grandissais, avec mon cousin, il m'abusait beaucoup. Donc, c'est pourquoi j'ai pas mal 
consommé, de la drogue et de l'alcool... Des abus sexuels, il me faisait faire des choses que je ne voulais pas faire... Il m'a dit de faire des choses que je ne voulais pas faire. Si je ne le faisais pas, il me battait. Il était plus vieux que moi, peut-être quatre ans de plus que moi. [...] Peut-être [quand j'avais] quatre ou trois ans, à peu près. [...] Je ne sais pas pourquoi je consommais, pour moi, je voulais quitter la réalité. Je voulais me geler tout le temps. (Philip, parent, 31 ans)

Parmi les préoccupations des Nunavimmiuts, le suicide est une question extrêmement délicate qui inquiète la population. Kenneth (2007) rapporte que la proportion de Nunavimmiuts ayant eu des idées suicidaires ou fait des tentatives de suicide a augmenté significativement passant de $12,5 \%$ en 1992 à plus de $20 \%$ en 2004. Le suicide est d'ailleurs aussi très préoccupant chez les Autochtones en général (Santé Canada, 2003 ; Poirier, 2005). Il serait de cinq à six fois plus élevé que chez les jeunes non-Autochtones (Santé Canada, 2003). Or, le suicide est cité par des répondants comme un événement qui intervient dans un parcours de consommation en provoquant une douleur psychologique qu'ils cherchent à oublier :

Mon fils le plus âgé a 20 ans. Il boit. Je ne lui en veux pas de boire. Cinq compagnons de classe avec qui il a grandi se sont tués. Ils se sont suicidés. Mon fils, il boit pour camoufler la souffrance. (Peggy, parent, 41 ans)

Korhonen (2004), ainsi que Dell et Lyons (2007), sont également d'avis que la consommation de SPA chez les Premières Nations, des Inuits et des Métis, peut devenir un mécanisme d'adaptation à des événements traumatisants, tel le suicide de proches.

Par ailleurs, beaucoup de familles inuites disposent de faibles revenus et le manque d'emploi contribue à cette situation économique. Craig fait référence ici à l'effet de la pauvreté sur les individus :

Alors, une autre motivation, c'est la pauvreté. Ce n'est pas facile de se débarrasser de la pauvreté ici. Il n'y a pas de moyen pour faire de l'argent. Il n'y a pas de mines, il n'y a pas de pétrole, personne ne «brasse des affaires» ici, vous savez... Alors, ils prennent de la drogue. Une certaine façon de s'échapper de la réalité. (Craig, leader, 54 ans)

Un deuxième niveau d'analyse permet d'entrevoir, en trame de fond, des causes de la consommation au Nunavik, une forme de normalité ou encore de fatalité:

C'est comme normal, ce n'est pas un problème. C'est juste comme normal, c'est la vie. (Julian, jeune, 17 ans) 
Je suis habitué, car tout le monde fume ou des fois les gens sont ivres dans la communauté. Selon moi, cela me paraît normal. Parce que je l'ai vu trop de fois. Les adolescents, veulent. Oui, je m'en fous s'ils fument, si tout le monde fume de la dope. Je m'en fous. C'est normal pour moi. Parce que [silence] je l'ai vu trop souvent. Depuis que je suis un enfant, tout le monde fume du pot. Pour moi, c'est juste normal. (Darryl, décrocheur, 17 ans)

Chansonneuve (2007) explique aussi que certains comportements de dépendance, initialement utilisés comme stratégies d'adaptation, se sont transformés en pratiques considérées normales et transmises de génération en génération. La transmission intergénérationnelle est d'ailleurs évoquée par plusieurs adultes et plusieurs jeunes de notre étude en tant que cause de l'usage abusif de SPA au Nunavik:

Quand je regarde ma mère qui boit comme... c'est tellement difficile de lui dire, de lui dire d'arrêter... l'alcool. Et même si je lui dis, elle va continuer quand même. Mon frère, mes frères et mes sœurs ils... ils sont presque comme alcooliques, mais je sens que je ne suis pas la seule à le faire dans ma famille. Je ne veux pas faire partie de leur consommation. (Katrina, jeune, 14 ans)

Quelques parents essaient d'arrêter leurs enfants de boire ou prendre de la drogue, mais quand ils sont trop stones ou trop ivres, ils commencent à leur donner de l'alcool ou de la drogue [...] c'est très difficile pour les jeunes parents d'apprendre comment être parents spécialement quand leurs parents le faisaient, ils pensent que c'est ok, donc ils le lèguent à leurs enfants. (Luke, leader, 25 ans)

Toujours dans le sens d'une forme d'imitation des comportements de consommation ou d'apprentissage social (Bandura, 1986) de ces comportements, plusieurs jeunes relatent également une soumission à une éventuelle pression des pairs à consommer :

Si tu ne fais pas de drogues, alors ils ne peuvent pas se tenir avec toi ou si t'en fais pas, t'es pas «cool», ou si t'en fais pas alors... tu vas juste être une mauviette pour le reste de ta vie. (Kathy, jeune, 16 ans)

J'ai été forcée par mes amis encore... ils continuaient de fumer, je faisais juste les regarder et eux ils essayaient de me le passer: «envoye, prends-le» et ils continuaient de me demander pour fumer alors... j'ai pris quelques "puffs». (Katrina, jeune, 14 ans)

À l'instar des autres Québécois, les Inuits consomment pour s'amuser, qu'il s'agisse des jeunes ou des adultes. Par ailleurs, l'ennui, l'adaptation au vécu difficile et la transmission intergénérationnelle émergent du 
discours des répondants comme des raisons importantes de la consommation de SPA des Nunavimmiuts. Mais ils évoquent aussi plusieurs conséquences de la consommation au Nunavik, qui seront maintenant rapportées.

\section{Conséquences de l'usage abusif de SPA}

Sur la question des conséquences de la consommation d'alcool et d'autres drogues, l'analyse du matériel permet de constater la présence dans le discours des répondants d'un large spectre de conséquences qui s'étalent sur un continuum de gravité subjectif pour chacun. Nous rapportons ici celles qui occupent le plus de place dans le discours des répondants et spécifions lorsque les adultes ou les jeunes en parlent davantage ${ }^{4}$. D'entrée de jeu, précisons que les jeunes de l'échantillon ont davantage parlé de violence, de problèmes à l'école et de disputes. Les adultes ont surtout évoqué la violence, les conséquences sur la famille, incluant l'appauvrissement, la question du syndrome d'alcoolisation fotale (SAF), la négligence et la mauvaise alimentation occasionnés selon eux par la consommation dans le village.

À travers plusieurs conséquences rapportées, les répondants ciblent la famille inuite comme une des victimes principales de la consommation. Parmi les effets de la consommation sur les relations familiales, plusieurs répondants font référence à l'infidélité et aux ruptures qu'elle entraîne dans beaucoup de situations. De leur point de vue, l'infidélité consécutive à l'intoxication provoque des bourrasques familiales qui font souffrir les enfants, lesquels ne sont pas dupes ni aveugles face à ces situations souvent très embarrassantes, surtout lorsque ces infidélités revêtent un caractère intrafamilial:

Lorsque nous sommes tous revenus dans notre salon, [pleure] il était assis en train de boire, [pleure] et il a dit: «Les enfants, je vous aime beaucoup.» Et j'ai dit: «Non tu ne nous aimes pas! Tu aimes [pleure] ton alcool, pas tes enfants!» Il a dit [pleure]: «Pourquoi?» [pleure] «Tu es toujours en train de tromper ta femme à cause de l'argent et de l'alcool, c'est ce que tu veux papa. [Pleure] Tu as une femme, tu ne l'aimes pas.» (Janice, jeune, 16 ans)

4. Autrement, nous utilisons l'appellation répondants pour refléter que tant des adultes que des jeunes en ont parlé. 
Violence, jalousie, infidélités, séparations sont autant de conséquences de la consommation pour la famille qui ne sont d'ailleurs pas propres aux Inuits (Brunelle et al., 2002). Les formes les plus fréquemment rapportées sont la violence conjugale et l'abus sexuel de la conjointe:

Alors les jeunes gars, ils se battent ou ils vont battre leur blonde. Ça leur donne plus de courage. Ils vont violer leur blonde ou ils vont la sodomiser quand ce n'est pas quelque chose qu' ils pourraient demander à leur blonde à faire à jeun, mais ils vont l'exiger quand ils sont saouls. Fait qu'il y a beaucoup d'abus. Les femmes en reçoivent beaucoup et les hommes en donnent beaucoup. (Lena, leader, 27 ans)

Browridge (2008) a d'ailleurs montré que les femmes autochtones risquaient de subir davantage d'abus physiques de la part de leur partenaire que les femmes non autochtones, et ce, surtout si ce partenaire agissait sous l'effet de l'alcool.

De plus, certaines femmes adultes de notre étude expliquent que les comportements d'infidélité et les relations sexuelles en état d'ébriété donnent naissance à des sentiments de honte et de culpabilité. Ils se doublent d'un sentiment de trahison envers la femme trompée et envers elles-mêmes également:

Il m’a amené jusqu'à la rivière et m’a fait l'amour là. J'étais sans défense. Je ne ferai jamais mal à sa femme, mais je l'ai fait. J'ai grandi avec elle, j'ai joué avec elle, je suis amie avec elle, mais j'ai brisé le lien parce que j'étais saoule. C'est la chose pour laquelle je suis vraiment fâchée. (Peggy, parent, 41 ans)

L'intoxication est aussi favorable à l'expression de la jalousie, laquelle entraîne à son tour la violence verbale et physique:

Pour moi, dans mes expériences, ce fut habituellement de m'accuser d'infidélité. M'accuser de voir un autre homme, d'avoir fait quelque chose ou de dire quelque chose dans son dos. Ce fut aussi de la suspicion, relativement à de la jalousie, cette violence verbale. (Cindy, leader, 50 ans)

D'autre part, la négligence à l'endroit des enfants, qui peut parfois découler de la consommation chez les parents, se manifeste de plusieurs façons. Les répercussions évoquées pour un enfant qui vit une situation de consommation à la maison peuvent être la fatigue, la faim, des problèmes de comportement. On peut aussi percevoir une certaine forme d'abandon: 
Après cela on joue aux cartes, on joue au bingo, on boit, on s'en prend l'un à l'autre, on ne s'occupe plus de ses enfants et ces derniers couraillent toute la nuit. C'était à cause de cela dans le passé, c'est comment ils étaient. [...] Nous jouions énormément aux cartes et nous les délaissions pour cela toute la nuit. (Alexander, Aîné, 63 ans)

Une autre forme de négligence souvent nommée par nos répondants est le manque de biens de base (nécessaires pour le soin des bébés, vêtements, etc.) et, plus particulièrement, une négligence alimentaire:

Le sniffing est venu d'un ami qui était très problématique et dont les parents étaient alcooliques et nos parents semblaient nous délaisser, et ils allaient dans les bars. Et nous nous tenions ensemble, comme des enfants sans endroit où aller. Restés à la maison, quelquefois nous avions faim... (Page, parent, 35 ans)

Ils n'ont pas de couches ou de serviettes humides pour bébés. Ou de la nourriture pour bébés à cause des drogues. C'est le problème comparativement à l'alcool. Et ils pourraient, à la place d'acheter un gramme à $50 \$$, ils pourraient avoir des couches, de la nourriture pour bébés, n'importe quoi pour le bébé, ils l'auraient à la place d'un gramme. (Preston, parent, 34 ans)

Plusieurs adultes nunavimmiuts parlent aussi d'un appauvrissement dû à la consommation de SPA, celle-ci pouvant entraîner des dépenses excessives ou la perte d'un emploi:

Plusieurs personnes qui ont des emplois, qui gagnent un salaire, ne sont pas capables de gérer ce revenu dans des choses positives comme acheter un nouveau canoë ou acheter une nouvelle motoneige ou des choses comme cela. Ils dévouent la majorité de leurs ressources pour nourrir leur dépendance. (Layne, leader, 54 ans)

Certains se départiront même du capital accumulé pour satisfaire cette nécessité ressentie de consommer:

Et nous vendons du vieux stock qu'on n'utilise plus, et certaines, j'ai vu certaines personnes finir avec plus rien, plus de TV, plus de divan, plus rien... aussi longtemps qu'ils boivent, ou fument... cela, cela me blesse parce que moi-même j'ai eu à vendre des choses pour supporter mon habitude. Et... ça fait mal de se séparer des choses que j’ai travaillé si fort pour avoir. (Marlen, parent, 34 ans)

L'endettement fait partie de ce cycle d'appauvrissement: «Maintenant, je dois aller au magasin et charger la dette. Et, certain jour quand nous 
sommes payés nous devons payer les dettes, un petit peu de marijuana » (Martin, parent, 29 ans).

Par ailleurs, un des effets de la consommation dans les familles, celle des parents, mais aussi des jeunes eux-mêmes, concerne les difficultés en milieu scolaire.

Je travaille à l'école, je peux remarquer les effets sur les enfants à l'école. Il y a beaucoup de comportements différents lorsque les familles sont affectées par l'alcool... La fatigue des enfants qui sont en retard, qui se sont couchés tard parce que leur famille buvait, la faim, qui affecte leur apprentissage et leur concentration, ainsi que leur travail. Ils sont très têtus, plus que les autres enfants, ils ne vont pas écouter le professeur. (Logan, leader, 48 ans)

Parmi les effets familiaux de la consommation de SPA au Nunavik, certaines femmes ont aussi évoqué le syndrome d'alcoolisme fotal (SAF) dans leur discours. Elles témoignaient d'une certaine conscience face aux dangers pour la santé du fotus que peut provoquer la consommation de SPA durant la grossesse:

J'étais consommatrice quand j'étais enceinte. Je ne sais pas... L'alcool peut avoir affecté, a eu un effet sur un de mes enfants c'est sûr, c'était aussi confirmé dans des rapports médicaux que j'ai reçus de l'école... Le rapport que j'ai eu, je sais qu'il a le SAF, pour son état mental je crois, je suis sûre parce que j'étais consommatrice à ce moment. (Marcy, parent, 43 ans)

À ce propos, Muckle et al. (2001) affirment que parmi un échantillon de 248 femmes enceintes du Nunavik, 60\% d'entre elles ont révélé avoir pris de l'alcool pendant leur grossesse et que la quantité moyenne d'alcool par occasion était de six verres. Près de la moitié des buveuses (49\%) se sont adonnées à des épisodes de consommation excessive «binge drinking», ce qui représente $30 \%$ des femmes enceintes étudiées. Or, de nombreuses recherches ont démontré les dangers reliés à la consommation d'alcool durant la grossesse (April et Bourret, 2004).

Enfin, notons que plusieurs conséquences de l'usage de SPA évoquées par les répondants (accidents, abus, violence...) sont reliées, dans leurs propos, principalement à l'usage d'alcool chez les Inuits, non pas à l'usage du cannabis ou d'autres drogues. En ce sens, Adlaf et PagliaBoak (2007) rappellent que chez les jeunes Canadiens, l'alcool est responsable de la plupart des méfaits graves liés aux habitudes de consommation (blessures, accidents). En termes de comportements déviants, l'alcool est souvent montré du doigt par les répondants de 
notre étude lorsqu'il est question de la conduite avec capacités affaiblies, de violence et de certains délits lucratifs. Les autres drogues sont surtout mises en cause dans le discours lorsque les répondants parlent de délits lucratifs tels que les vols et la prostitution.

Pour ce qui est de la conduite avec capacités affaiblies, les répondants relatent des situations impliquant des voitures, mais aussi des bateaux, des motoneiges et des véhicules tout-terrain. Ces véhicules sont souvent utilisés pour des sorties de chasse ou de pêche sur le territoire, contextes qui comportent leur lot de dangers:

Beaucoup, vraiment beaucoup sont morts depuis que l'usage de l'alcool et des stupéfiants est conjugué avec ces motoneiges et ces véhicules toutterrain. (Elias, Aîné, 70 ans)

Mon petit-fils, qui devrait être en vie aujourd'hui, était un consommateur d'alcool et il est mort à cause de cela; il était parti à la chasse au morse en bateau alors qu'il était en boisson et il s'est noyé. Alors que beaucoup de personnes pourraient continuer à vivre, l'alcool peut les conduire jusqu'à la mort. (Anthonny, Aîné, 94 ans)

Ces accidents qui arrivent sur le territoire sont un sujet de préoccupation dans les villages et des messages radio sont diffusés sur la nécessité de ne pas consommer lorsque l'on se déplace sur le territoire:

Ils annoncent toujours sur le FM de ne pas prendre de l'alcool et des drogues quand nous allons pêcher ou chasser. Parce qu'il y a des gens qui sont morts ou ont eu des accidents. (Peter, parent, 35 ans)

Par ailleurs, la violence extra-familiale comme conséquence de la consommation est très présente dans les discours. En effet, les bagarres entre individus intoxiqués, par l'alcool le plus souvent, sont évoquées: «Il y a un de mes amis qui a été battu à mort par un saoul» (Marlen, parent, 34 ans). Cette crainte du meurtre est d'ailleurs directement nommée: «C'est un très grave problème car cela peut mener au meurtre» (Anthony, Aîné, 94 ans).

Plusieurs répondants révèlent que le vol est un acte délinquant assez commun chez les consommateurs pour financer leur consommation. Ils volent de l'argent, des objets ou directement des substances:

Voler est quelque chose qui se produit lorsque les gens ont beaucoup d'argent dans leurs poches et qu'ils perdent connaissance, autant l'argent, la bouteille ou la drogue. Lorsque quelqu'un en a l'occasion, ils vont voler. (Cindy, leader, 50 ans) 
Enfin, comme ailleurs, il semble que certaines femmes du Nunavik négocient du sexe contre de l'alcool ou du cannabis ou encore de l'argent pour s'en procurer:

Les filles ont beaucoup moins de misère à trouver de l'argent parce qu'elles font une sorte de prostitution, elles peuvent se prostituer pour en avoir. (Lena, leader, 27 ans)

En termes de déviance, plusieurs des propos des répondants sur les conséquences de la consommation de SPA au Nunavik reflètent de populaires modèles théoriques explicatifs des liens drogue-crime. D'abord, on trouve le modèle psychopharmacologique selon lequel les SPA induisent des comportements violents chez les individus intoxiqués (Goldstein, 1985). Aussi, le modèle économico-compulsif est présent dans le discours (Nurco et al., 1991). Celui-ci explique la délinquance lucrative chez les personnes alcooliques ou toxicomanes par le fait qu'elles ne peuvent pas assumer financièrement les dépenses qu'occasionne leur dépendance. Brochu (2006) explique toutefois que d'autres modèles explicatifs des liens drogue-crime existent et que la nature de ces liens évolue au fil de la trajectoire des consommateurs.

Les répondants ont beaucoup parlé de liens qu'ils perçoivent entre des comportements délinquants ou déviants tournés contre ou vers autrui (différentes formes d'agression, de délits lucratifs) et la consommation de SPA, mais plusieurs ont aussi parlé de comportements autodestructeurs induits par l'intoxication, particulièrement le suicide. Certains croient que des personnes de leur village se sont suicidées sous l'influence de SPA ou même en raison du manque de substances (craving, sevrage):

Je n'étais pas avec lui quand c'est arrivé, mais c'est la même soirée, nous sniffions ensemble et nous nous tenions ensemble, il n'arrêtait pas de dire «je vais me tuer», mais il ne voulait pas que je m'en aille parce qu'il allait juste s'embrouiller... Et le lendemain, j'ai entendu dire qu'il s'était tué. (Judy, décrocheuse, 16 ans)

Wexler (2006) explique que la consommation d'alcool est impliquée dans la plupart des cas de suicide chez les Inuits. Katz et al. (2006) rapportent que les problèmes de consommation de SPA constituent un facteur de risque relié au suicide. Le manque de stratégies d'adaptation productives (Pronovost et al., 2003) reliées aux problèmes de toxicomanie entraîne parfois des intentions suicidaires ou des passages à l'acte. 


\section{Conclusion}

Les répondants nunavimmiuts de notre étude évoquent différentes raisons pour expliquer la consommation de SPA au Nunavik. Les jeunes insistent sur des raisons un peu différentes de celles des adultes. Ces derniers étant beaucoup plus volubiles au sujet de la colonisation et de ses effets, dont fait partie l'usage abusif d'alcool et d'autres drogues. Les conséquences de la consommation évoquées concernent beaucoup les familles et les comportements déviants. Les adultes mentionnent davantage les questions d'appauvrissement, de SAF et de négligence envers les enfants que les jeunes, qui parlent aussi de problèmes scolaires. Les répondants jeunes et adultes parlent beaucoup de situations de violence qui sont occasionnées selon eux par la consommation de SPA au Nunavik.

Mais les problèmes que vivent les Inuits sont-ils nécessairement et entièrement causés par l'alcool ou les autres drogues? Par exemple, l'usage excessif d'alcool allié à une grande proximité ou promiscuité entre les gens peut amplifier les risques d'abus de toutes sortes dans les villages inuits. Lorganisation Inuit Tapiriit Kanatami (2004) explique que cette situation représente un risque pour la santé physique et psychosociale des Nunavimmiuts. Dans l'étude de l'INSPQ conduite au Nunavik en 2004, certains répondants ont notamment proposé d'augmenter la quantité de logements disponibles pour éviter que trop de personnes vivent sous le même toit et ainsi réduire la violence (Lavoie et al., 2007). Il n'est pas facile de départager ce qui est associé à (ou «causé par») la consommation de ce qui relève d'autres facteurs indépendants. Brownridge (2008) par exemple explique que la violence conjugale chez les Autochtones n'est pas uniquement due à la consommation de substances, mais elle s'explique aussi par la colonisation et les sentiments de dépossession qui provoquent des émotions négatives à l'origine du passage à l'acte. Il est toutefois certain que les problèmes de consommation occasionnent de la détresse psychologique chez les Inuits (Kenneth, 2007), ce qui n'aide pas à améliorer leurs conditions de vie biopsychosociales.

Plus globalement, mentionnons que certains thèmes ont été évoqués par les répondants à la fois en termes de causes et de conséquences de la consommation de SPA dans les villages du Nunavik. Des problèmes d'emploi, de pauvreté, de violence, d'abus sexuel et de suicide ont été vus en tant que causes mais également comme des conséquences de 
l'abus de substances. Un cercle vicieux tend à se dessiner dans les villages inuits, en particulier quand ces problèmes sont présents. La grande place qu'occupe la souffrance ou le mal-être dans ces villages transpire dans les propos des répondants: histoire ou colonisation, abus sexuel et physique, suicide, et laisse entrevoir un cercle vicieux ou du moins des frontières floues entre causes et conséquences de la consommation qu'on y trouve. En ce sens, Korhonen (2004: 31) écrivait: «Drinking has thus become both a source of problems and a strategy for coping with problems, especially those associated with social dislocation and economic marginalization.»

La pertinence sociale de cette étude consiste à fournir aux Nunavimmiuts des éléments de compréhension de la consommation abusive de SPA au Nunavik pour conduire à des solutions adaptées à leur situation. Ce propos fait d'ailleurs l'objet d'un article dans la prochaine parution de la revue Drogues, santé et société (Plourde et al., soumis).

\section{Références}

Adlaf, E. M., \& Paglia-Boak, A. (2007). Drug use among Ontario students: findings from the OSDUS, 1977-2007. Toronto: Centre for Addiction and Mental Health.

Anctil, M. (2008). Enquête de Santé auprès des Inuit du Nunavik 2004: Les faits saillants de l'enquête. Qanuippitad? Comment allons-nous? Québec et Kuujjuak: Institut national de santé publique du Québec / Régie régionale de la santé et des services sociaux du Nunavik.

April, N., \& Bourret, A. (2004). État de situation sur le syndrome d'alcoolisation foetale au Québec. Québec: Institut national de santé publique du Québec.

Bandura, A. (1986). Social Foundation of Thought and Action: A Social Cognitive Theory. Englewood Cliff: Prentice-Hall.

Brochu, S. (2006). Drogues et criminalité: Une relation complexe ( $2^{\mathrm{e}}$ éd.). Montréal: Les Presses de l'Université de Montréal.

Brownridge, D. A. (2008). Understanding the Elevated Risk of Partner Violence Against Aboriginal Women: A Comparison of Two Nationally Representative Surveys of Canada. Journal of Family Violence, 23, 353-367.

Brunelle, N., Cousineau, M.-M., \& Brochu, S. (2002). La famille vécue par des jeunes consommateurs de drogues et trajectoires types de déviance juvénile. Drogues, santé et société, 1 (1), 82-103.

Brunelle, N., Cousineau, M.-M., \& Brochu, S. (2005). Juvenile Drug Use and Delinquency: Youths' accounts of their trajectories. Substance Use and Misuse, 40 (5), 721-734.

Brunelle, N., Plourde, C., Landry, M., Gendron, A., Mercier, C., \& Eveno, S. (sous presse). Patterns of Psychoactive Substance Use among Youth in Nunavik. Dittera. 
Chansonneuve, D. (2007). Addictive Behaviours Among Aboriginal People in Canada. Ottawa: Aboriginal Healing Foundation. (En ligne). http://www.ahf.ca/ publications/research-series.

Dell, C. A., \& Lyons, T. (2007). Harm reduction policies and programs for persons of Aboriginal descent. Ottawa: Canadian Centre on Substance Abuse.

Deslauriers, J. P. (1991). Recherche qualitative. Guide pratique. Montréal: Chenelière/McGraw-Hill.

Dufort, F., Le Bossé, Y., \& Papineau, D. (2005). La recherche en psychologie communautaire: La construction des connaissances à travers l'action. In F. Dufort \& J. Guay (Eds.), Agir au cour des communautés. La psychologie communautaire et le changement social (369-408). Ste-Foy: Les Presses de l'Université Laval.

Fletcher, A. C., \& Hunter, A. G. (2003). Strategies for obtaining parental consent to participate in research. Family Relations, 52 (3), 216-221.

Giorgi, A. (1997). De la méthode phénoménologique utilisée comme mode de recherche qualitative en sciences humaines: théorie, pratique et évaluation. In J. Poupart, J. P. Deslauriers, L. H. Groulx, A. Laperrière, R. Mayer \& A. Pires (Eds.), La recherche qualitative. Enjeux épistémologiques et méthodologiques (341-390). Montréal: Gaëtan Morin Éditeur.

Goldstein, P. J. (1985). The Drugs/Violence Nexus: A Tripartite Conceptual Framework. Journal of Drug Issues, 14, 493-506.

Greabell, L., Cordes, P., \& Klein, S. J. (2005). HIV/AIDS and Native Americans: The Health Departments' Response. Journal of Psychoactive Drugs, 37 (3), 267-272.

Huot, F. (2007). Un Ivoirien chez les Innuvialuits : réflexions sur l'intervention sociale dans les Territoires du Nord-Ouest. Entrevue avec Kouakou Fiendi Pira, intervenant au Centre de santé et de services sociaux de Paulatuk, Territoires du Nord-Ouest (Canada). Nouvelles pratiques sociales, 20 (1), 29-38.

Inuit Tapiriit Kanatami (2004). 5000 Years of Inuit History and Heritage. Consultée le 8 mai 2009 , http://www.itk.ca/sites/default/files/5000YearHeritage.pdf.

Katz, L. Y., Elias, B., O’Neil, J., Enns, M., Cox, B. J., Belik, S.-L., \& Sareen, J. (2006). Aboriginal suicidal behaviour research: From risks factors to culturally-sensitive interventions. Journal of the Canadian Academy of Child and Adolescent Psychiatry, 15 (4), 159-167.

Kenneth, P. (2007). Correlates of Psychological Distress Amongst Adult Inuit in Nunavik, Northern Quebec. Mémoire, Université McGill, Montréal.

Korhonen, M. (2004). Alcobol Problem and Approaches: Theories, Evidence and Northern Practice. Ottawa: National Aboriginal Health Organization (NAHO). Consulté le 29 mars 2006, http://www.naho.ca/english/pdf/alcohol_problems_approaches.pdf.

Lavoie, F., Fraser, S., Boucher, O., \& Muckle, G. (2007). Prevalence and nature of received sexual violence in Nunavik. In D. St-Laurent, É. Dewailly \& S. Déry (Eds.), Nunavik Inuit bealth survey 2004: Qanuippitad? How are we? Québec: Institut national de santé publique du Québec et Régie régionale de la santé et des services sociaux du Nunavik. 
Mercier, C., Rivard, J., Guyon, L., \& Landry, M. (2002). Consommation d'alcool et de drogues dans les communautés du Nunavik: Les données épidémiologiques et des problèmes associés. Tome I et II. Rapport de la Phase I adressé à la Régie régionale de la santé et des services sociaux du Nunavik.

Muckle, G., Ayotte, P., Dewailly, E. E., Jacobson, S. W., \& Jacobson, J. L. (2001). Prenatal Exposure of the Northern Quebec Inuit Infants to Environmental Contaminants. Environmental Health Perspectives, 109 (2), 1291-1292.

Nurco, D. N., Hanlon, T. E., \& Kinlock, T. W. (1991). Recent Research on the Relationship between Illicit Drug Use and Crime. Behavioral Sciences $\mathbb{F}$ the Law, 9, 221-242.

Paillé, P., \& Mucchielli, A. (2003). L'analyse qualitative en sciences bumaines et sociales. Paris: Armand Colin.

Plourde, C., Brunelle, N., Landry, M., Guyon, L., Mercier, C., Gendron, A., \& Eveno, S. (2007). Consommation de substances psychoactives chez les jeunes du Nunavik: résultats de l'enquête. Rapport synthèse \# 1 présenté à 1'Institut de recherche en santé du Canada (IRSC) et à la Régie régionale de la santé et des services sociaux du Nunavik.

Plourde, C., Brunelle, N., \& Landry, M. (soumis). Faire face à l'usage de substances psychoactives chez les Inuit du Nunavik: Pratiques nordiques et points de vue de Nunavimiuts sur la question. Drogues, santé et société.

Poirier, J-G. (2005). Le suicide chez les Atikamekw versus les autres nations. Conseil de la nation Atikamekw.

Poupart, J., Deslauriers, J.-P., Groulx, L.-H., Laperrière, A., Mayer, R., \& Pires, A. P. (1997). La recherche qualitative: Enjeux épistémologiques et méthodologiques. Montréal: Gaëtan Morin.

Pronovost, J., Leclerc, D., \& Dumont, M. (2003). Facteurs de protection reliés au risque suicidaire chez des adolescents: Comparaison de jeunes en milieu scolaire et de jeunes en Centres jeunesse. Revue québécoise de psychologie, 24 (1), 179-199.

Régie régionale de la santé et des services sociaux du Nunavik (2004-2005). Rapport annuel 2004-2005. Consulté le 20 février 2006, http://www.rrsss17gouv.qc.ca/data/rapports_annuels/RRSSSN_Rapport Annuel_Francais_2004_2005-3.pdf.

Saewyc, E. (2007). La consommation de substances chez les jeunes marginalisés. In Centre canadien de lutte contre l'alcoolisme et les toxicomanies (Ed.), Toxicomanie au Canada: Pleins feux sur les jeunes (14-21). Ottawa: Centre canadien de lutte contre l'alcoolisme et les toxicomanies.

Santé Canada (2003). Acting on What we Know: Preventing Youth Suicide in First Nations. Ottawa: Santé Canada.

Shaggers, S., \& Gray, D. (2002). Explanations of Indigenous Alcohol Use. In D. Gray and S. Shaggers (Eds), Indigenous Australian Alcobol and Other Drug Issues: Research from the National Research Institute (169-191), Perth : National Drug Research Institute, Curtin University of Technology. 
Statistique Canada (2006). Recensement de 2006 : Peuples autochtones du Canada en 2006: Inuit, Métis et Premières nations. Recensement de 2006 : résultats. Consulté le 21 juin 2007 , http://www12.statcan.ca/francais/census06/analysis/aboriginal/Inuit. cfm.

Wexler, L. M. (2006). Inupiat Youth Suicide and Culture Loss: Changing Community Conversations for Prevention. Social Science \& Medicine, 63, 2938-2948.

ABSTRACT - Results from a study on the use of psychoactive substances in Nunavik are presented. The qualitative part of the study consisted of 108 semi-structured qualitative interviews with youth, parents, leaders and elders of four villages. Thematic content analysis of the material revealed different reasons and consequences of substance use and abuse in Nunavik, from the respondent's standpoint. Among the reasons, respondents evoked pleasure, boredom and lack of activities, "colonization," coping about different problems (poverty, victimization and suicide) and also intergenerational transmission. Concerning consequences, they mostly talked about family impacts including intimate violence and child neglect, but also about other deviant behaviors like drinking and driving and lucrative crimes. These results are discussed according to actual knowledge about causes and consequences of substance use among Inuit and non-Inuit.

KEYWORDS - Drugs, Alcohol, Inuit, Nunavik, Quebec, qualitative research.

RESUMEN - Este artículo presenta los resultados de un estudio sobre el consumo de sustancias psicoactivas en Nunavik. El estudio incluye 108 entrevistas cualitativas semidirigidas con jóvenes, padres de familia, líderes y ancianos de cuatro poblaciones. El análisis del contenido temático de los resultados mostró diferentes razones y diversos efectos del consumo de dichas sustancias, según los participantes. Entre las razones evocadas se mencionan el placer, el aburrimiento y la falta de actividades, la "colonización", la necesidad de enfrentar diversos problemas (la pobreza, el abuso, el suicidio) y la transmisión intergeneracional. Respecto de las consecuencias del consumo, se mencionaron principalmente los impactos sobre la familia, por ejemplo la violencia conyugal y la negligencia de los niños, además de comportamientos desviantes como el conducir con facultades disminuidas, las agresiones y los delitos con lucro. Estos resultados se evalúan considerando los conocimientos actuales sobre las causas y las consecuencias del consumo tanto en los Inuit como en la población general.

PALABRAS CLAVE • Droga, alcohol, inuit, Nunavik, Quebec, investigación cualitativa. 